\title{
Numerical studies of an interacting particle system and its deterministic description
}

\author{
Emilio Hernández-García and Cristóbal López \\ Instituto Mediterráneo de Estudios Avanzados IMEDEA (CSIC-UIB), Campus de \\ la Universitat de les Illes Balears, E-07122 Palma de Mallorca, Spain. ${ }^{1}$
}

\begin{abstract}
We present numerical results on a stochastic population dynamics model with reproduction rates depending on the density of particles in a neighborhood. In order to investigate the role of fluctuations some characteristics of the model are compared with those computed from its deterministic (noise-less) density equation. In particular, we study the stationary density of particles when a pattern is developed and the typical size of the clusters of particles.
\end{abstract}

Key words: Interacting particle systems, Cluster formation, Pattern formation

\section{Introduction}

Interacting particle systems mimicking biological individuals competing for resources may provide insights into the dynamics of real populations. Aiming at this, the authors recently introduced [1] a simple model of particles representing bugs that reproduce and die at rates depending on the number of particles present within a given distance. The main feature of the model is that, for a specific range of the parameters, the particles arrange in a periodic pattern of clusters. In previous works $[1,2]$ it was shown that this can be explained as an instability in the deterministic equation derived for the density of particles. However, some of the properties of the original particle system cannot be explained within the framework of a deterministic density equation because of the fluctuations arising from the discrete character of the particles. Here we briefly present further properties of the model and of the deterministic description that properly establishes the role of fluctuations. Considering the

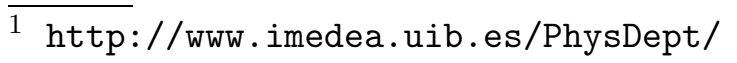


rather general mechanism introduced in the particle interaction (competition with neighbors), the implications of these studies can be extended to more realistic biological population dynamics.

The model consists of $N(t)$ particles at time $t$ performing Brownian motion, with diffusion coefficient $D$, in the line (we restrict in this paper to the onedimensional periodic case: a ring of length $L)$. Each particle $i(=1, \ldots, N)$ reproduces with rate (probability per unit of time) $\lambda_{i}$, giving rise to a newborn which is located at the same place that the parent particle, or dies at rate $\beta_{i}$, disappearing from the system. The death rate $\beta_{i} \equiv \beta_{0}$ is a constant and the same for all the particles, but the birth rate $\lambda_{i}$ models competition for resources, so that it decreases with the number of neighbors $N_{R}^{i}(t)$ that particle $i$ has within a given distance $R: \lambda_{i}=\lambda_{0}-N_{R}^{i}(t) / N_{s} . \lambda_{0}$ and $N_{s}$ are constants. In Ref. [1] the following equation for the expected density of particles $\phi(x, t)$ was derived under the approximation of negligible fluctuations:

$$
\partial_{t} \phi(x, t)=\mu \phi(x, t)+D \nabla^{2} \phi(x, t)-\frac{1}{N_{s}} \phi(x, t) \int_{|x-y| \leq R} \phi(y, t) d y,
$$

where $\mu=\lambda_{0}-\beta_{0}$. This deterministic integrodifferential equation and similar ones have been directly introduced in related contexts $[3,4]$. For $\mu>\mu_{P}=$ $84.2 D / R^{2}$ its solution tends at long times to a steady spatially periodic pattern, which explains the clustering occurring in the particle system.

In this Paper we summarize some numerical and analytical calculations of the stationary density of particles (Sect. 2), and the size of clusters (Sect. 3). In both cases, the emphasis is in the comparison of the behavior of the stochastic particle model with the deterministic density approximation (1).

\section{Stationary density of particles}

Figure 1 shows (circles) the mean density of particles (defined as the temporal average $\rho$ in the quasisteady state of $\rho(t)=N(t) / L$, the quotient between the total number of particles $N(t)$ and system size $L)$. There is a sharp transition between the absorbing state $\rho=0$ and the active phase $\rho>0$ when increasing the parameter $\mu=\lambda_{0}-\beta_{0}$ above a critical value $\mu_{c}(\approx 0.34$ in the figure). At sufficiently large values of $\mu$ the density approaches a nearly linear dependence on $\mu$. The transition behavior given by the numerical solution of the deterministic model (1) is completely different (squares), as stressed in [2]. In particular the deterministic prediction indicates that the active phase appears above $\mu_{c}=0$, which is much below the particle-model $\mu_{c}$. In fact, $\mu_{c}$ is above the deterministically predicted value for $\mu_{P}\left(\mu_{P} \approx 0.084\right.$ for the parameters 
of the figure), consistent with the observation that the active phase in the particle simulations already displays a spatially periodic character immediately above threshold. In contrast to these inaccuracies close to the transition region, $\rho$ closely approaches the deterministic result at larger $\mu$.

In addition to the numerically determined value of $\rho$ obtained from (1), one can also obtain analytical expressions for it that better clarify the processes involved. For example, one can compare the homogeneous solution of (1), $\rho_{0}=\mu N_{s} /(2 R)$ with the true $\rho$ in the pattern state. Figure 1 shows that $\rho_{0}$ is below the pattern value. A way to understand this consequence of the pattern instability is to evaluate the effect of the periodic modes into the homogeneous one due to their nonlinear interactions. This can be done perturbatively close enough to the pattern forming instability point $\mu \approx \mu_{P}$ by pushing one order further the amplitude calculation of reference [2]. The result is that there is a correction at order $\mu-\mu_{p}$ to the average density $\rho=L^{-1} \int_{L} d x \psi(x)$ :

$$
\begin{array}{ll}
\rho=\frac{\mu N_{s}}{2 R}, & \text { if } \mu<\mu_{P} \\
\rho=\frac{\mu N_{s}}{2 R}+\gamma \frac{\left(\mu-\mu_{P}\right) N_{s}}{2 R}, & \text { if } \mu>\mu_{P}
\end{array}
$$

where $\gamma \approx 0.2605$. This expression for $\rho$ is plotted as a dashed line in Fig. 1 where one can see that it provides a good approximation to the behavior at large $\mu$, where the stochastic and the deterministic models agree. Moreover, the inset in Fig. 1 also shows that (2) is an accurate description of the deterministic mean density close to the deterministic pattern forming instability. The calculation leading to (2) highlights that the reason for the increased $\rho$ with respect to the homogeneous value $\rho_{0}$ is the nonlinear interaction between the homogeneous and the pattern forming mode.

A more intuitive and simple argument to obtain an approximation to $\rho$ consists in recognizing that the average density is the number of particles per cluster $N_{1}$ divided by the distance between clusters. The linear analysis of the pattern onset from $(1)$ gives this distance to be $f R$, with $f \approx 1.54$. Thus, only particles in the same cluster contribute to the neighborhood of a given particle $i$, and $N_{R}^{i}=N_{1}$. The number of particles $N_{1}(t)$ in each cluster will stabilize when the net growth rate felt by any of its particles $\lambda_{0}-\beta_{0}-N_{1} / N_{s}$ becomes close to zero, so that $N_{1} \approx \mu N_{s}$. The numerical result indicates that $N_{1}$ fluctuates close to, but in average slightly below, that value. Dividing this estimation for $N_{1}$ by the distance between clusters one gets

$$
\rho=\frac{\mu N_{s}}{f R}
$$

This expression turns out to be accurate in the whole range of $\mu$ studied (for 


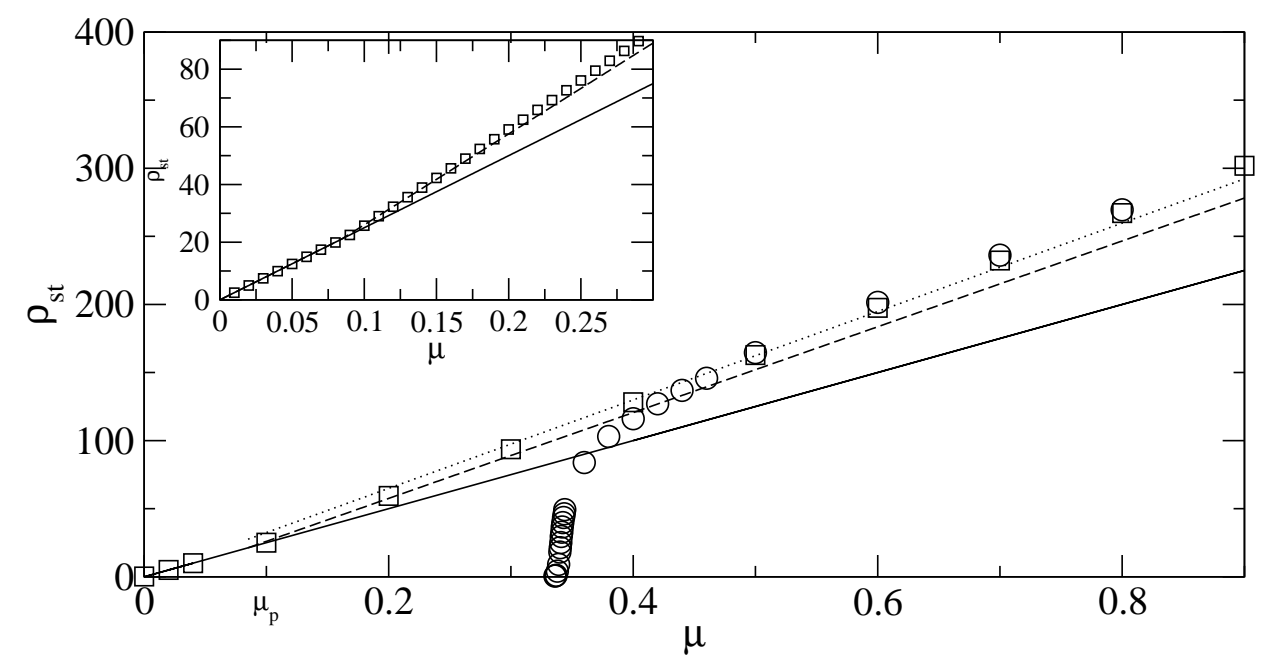

Fig. 1. Stationary mean density versus $\mu$. Circles are for the stochastic particle model and squares for the deterministic density description. Solid line corresponds to the homogenous density $\rho_{0}$, dashed line to the perturbative calculation (2), and dotted line to the approximation (3) with $f=1.54$. In the inset we focus on values of $\mu$ close to $\mu_{p} \approx 0.084$. Again squares are from the deterministic equation, solid line is $\rho_{0}$, and dashed line is (2). $D=10^{-5}, N_{s}=50, R=0.1$ and in the particle model we change $\mu$ while keeping $\lambda_{0}+\beta_{0}=1$.

$D$ small enough to guarantee clustering) as Fig. 1 shows (dotted line).

\section{Size of the clusters}

In this section we report calculations on the typical size, $S_{c}$, of the clusters appearing in the system. Heuristically, we can argue that particles inside a cluster will diffuse a distance of the order of $\sqrt{2 D \tau}$ during a time $\tau$. Since the mean time to death is $\beta_{0}^{-1}$, it is natural to expect the particles to be confined in clusters of size $S_{c} \propto \sqrt{D / \beta_{0}}$. In the simulations of the particle model the typical cluster size was calculated by averaging the sizes (rms dispersion) of all the clusters in the system at a given time, and then averaging for many (6000) time steps in steady state conditions. In the deterministic model, the typical size of a cluster is calculated as the width of a hump in $\phi$ (in stationary conditions all the humps are identical) at the semi-peak height. For different values of the diffusion coefficient and fixed $\mu=0.6$ both measurements are plotted in the left panel of Fig. 2. The best straight line fitted to the particle data has slope $1 / 2$, thus confirming the expected diffusive dependence of cluster size, i.e., $S_{c} \propto \sqrt{D}$. This behavior of the discrete system is in contrast with the deterministic density approximation, where the size of the humps only changes slightly with the diffusion coefficient. Hence it is obvious that fluctuations and 

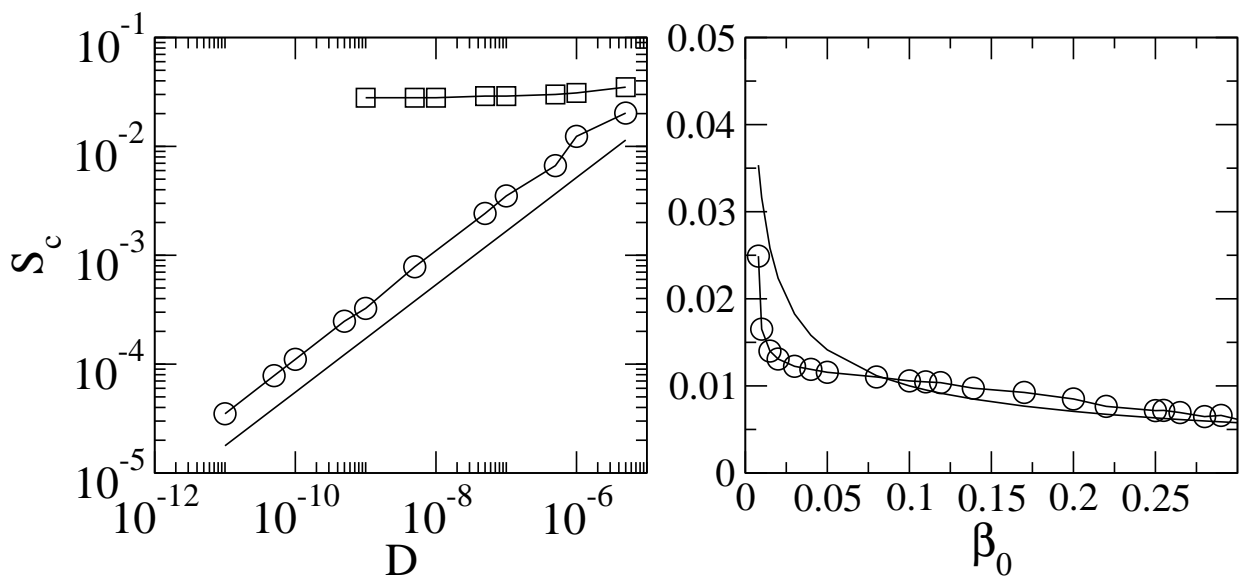

Fig. 2. Left: size of clusters vs diffusion coefficient for $\lambda_{0}=0.8, \beta_{0}=0.2$, so that $\mu=0.6$. Squares are from (1) and circles from particle simulations. The straight line has slope $1 / 2$, the best fit to the particle data. Right: size of clusters vs death rate $\beta_{0}$ from particle simulations (circles). $D=10^{-5}$ and $\lambda_{0}=1-\beta_{0}$. The line is the function $S_{c}=\sqrt{10 D / \beta_{0}}$. In both panels $R=0.1$ and $N_{s}=50$.

the discrete character of the particles play a fundamental role.

In the right panel of Fig. 2 we plot $S_{c}$ versus the death rate $\beta_{0}$. As predicted by the heuristic argument, cluster size increases when $\beta_{0}$ decreases, but the precise prediction $S_{c} \propto \sqrt{D / \beta_{0}}$ is only qualitatively correct, so that processes other than the simple particle death are influencing cluster size.

We believe that the different behavior of the stochastic and the deterministic model for the cluster size arises, as the differences in the transition region for the mean density, from the absorbing [5] character of the state empty of particles, or $\phi=0$, which is not captured when the multiplicative noise inherent to particle reproduction is ignored. Further work to clarify this issue is clearly needed.

Financial support from MEC (Spain) and FEDER through project CONOCE2 (FIS2004-00953) is greatly acknowledged. C.L. is a Ramón y Cajal fellow.

\section{References}

[1] E. Hernández-García and C. López, Phys. Rev. E 70 (2004), 016216.

[2] C. López and E. Hernández-García, Physica D 199 (2004) 223-234 .

[3] M.A. Fuentes, M.N. Kuperman, V.M. Kenkre, Phys. Rev. Lett. 91 (2003) 158104. 
[4] A. Sasaki, J. Theor. Biol. 186 (1997) 415-430.

[5] G. Grinstein, M.A. Muñoz, in Fourth Granada Lectures in Computational Physics, P.L. Garrido and J. Marro editors (Springer, Berlin, 1997). 